\title{
«ENTONCES USTED NIEGA ¿NO? ¿O QUE NO SE ACUERDA?»: ESTUDIO CUANTITATIVO DE LOS TIPOS DE PREGUNTAS UTILIZADAS EN INTERROGATORIOS JUDICIALES ESPAÑOLES*
}

\author{
«ENTONCES USTED NIEGA ¿NO? ¿O QUE NO SE ACUERDA?»: QUANTITATIVE \\ STUDY OF TYPES OF QUESTIONS USED IN SPANISH JUDICIAL EXAMINATIONS
}

\author{
YolANDA RUANO LAPARRA \\ yolanda.ruano@um.es \\ Susana Ridao RodRigo \\ sridao@um.es \\ Universidad de Murcia
}

Recibido: 14/03/2016

Aceptado: 19/07/2016

\begin{abstract}
Resumen
Esta investigación se centra en analizar los tipos de preguntas utilizadas en interrogatorios judiciales españoles. Para ello, se han analizado dos corpus de juicios orales celebrados en dos zonas geográficamente dispares de España: Almería y Barcelona. En el presente artículo se argumenta, con datos estadísticos, el frecuente uso de enunciados con fines interrogativos cuya entonación responde a patrones prototípicos aseverativos; esta tipología presenta mayores índices de frecuencia en el corpus recogido en Almería (43.9\%) que en el de Barcelona (23.7\%).

PALABRAS CLAVE: lingüística forense, pragmática, interrogatorios judiciales, interrogaciones, entonación.

Abstract

The aim of this research is to analyze the types of questions used in Spanish judicial interrogation. For this, two corpus of oral trials taken place in two geographically different areas of Spain have been analyzed: Almería and Barcelona. The present article argues, with statistics data, the frequent use of sentences with interrogative intentions whose intonation is prototypical assertive patterns; this type has higher rates in the corpus collected in Almería $(43.9 \%)$ than in Barcelona (23.7\%).

KEYWORDS: Forensic Linguistics, Pragmatics, judicial examinations, questions, intonation.

* Originariamente este artículo tenía como objetivos tanto el análisis cuantitativo como el cualitativo e iba a ser publicado con Ángel Cervera y José Torres, ambos investigadores vinculados a la Universidad Complutense de Madrid. Cuando se trabajó en el análisis, se observó que la extensión de ese primer manuscrito sobrepasaba bastante los parámetros estándares de los artículos de investigación, de ahí que se decidiera dividir en dos publicaciones: la dimensión cualitativa había sido trabajada por Ángel Cervera y José Torres y la dimensión cuantitativa por las autoras que firman la presente investigación. Aprovechamos la ocasión para agradecerles encarecidamente a estos profesores los consejos recibidos. También queremos mostrar nuestro más sincero agradecimiento a los evaluadores de este artículo, puesto que sus sugerencias han permitido mejorar notoriamente la presente investigación.
\end{abstract}

Para citar este artículo / To cite this article: Ruano Laparra, Yolanda; Ridao Rodrigo, Susana (2016). «Entonces usted niega ¿no? ¿O que no se acuerda?»: estudio cuantitativo de los tipos de preguntas utilizadas en interrogatorios judiciales españoles. ELUA, 30: 239-255. doi:10.14198/ELUA2016.30.11

Enlace / Link: http://dx.doi.org/10.14198/ELUA2016.30.11 


\section{Introducción}

Desde tiempos pretéritos el sometimiento de un sujeto a un interrogatorio ha transmitido connotaciones bastante negativas. De hecho, ya el mismo Miguel de Cervantes en el capítulo quinto de la primera parte de El Ingenioso Hidalgo don Quijote de la Mancha ponía de manifiesto la hostilidad del protagonista al asumir el rol de interrogado, como puede verse en el siguiente fragmento: "Hizieronle a don Quixote mil preguntas, y a ninguna quifo refponder otra cofa, fino que le dieffen de comer, y le dexaffen dormir, que era lo que mas le importaua" (Cervantes 1605: 15). La tercera acepción del Diccionario de la Lengua Española señala que un interrogatorio es el "Acto de dirigir una serie de preguntas a quien las ha de contestar” (RAE-ASALE 2014). En esta definición queda sobrentendida la jerarquía existente: interrogador e interrogado. Quizá sea esta disparidad implícita de escalafón lo que motive la mala prensa otorgada a dicho vocablo.

Centrados en el campo del interrogatorio judicial, cabe preguntarse por qué los estudios sobre el discurso judicial con corpus orales han aparecido de manera tan tardía, sobre todo en el ámbito hispánico frente al anglosajón. Es cierto que en los últimos años están despertando bastante interés dichos trabajos, fundamentalmente desde el enfoque de la traducción y la interpretación, como consecuencia de ser conscientes de la incuestionable relevancia que en los juicios orales asume el mensaje emitido por cada participante. Diversas investigaciones señalan que el gran óbice con el que se encuentran estos estudios radica en la dificultad en la obtención del corpus. Más exactamente, “[...] owing to severe restrictions on research access to Crown Courts, there has been virtually no study of the actual language used in English jury trials" (Heffer 2005: xvii).

A la par, en el contexto español, Taranilla observa que un obstáculo principal en el acceso a este tipo de corpus es la privacidad de los temas tratados en los entornos penales; igualmente, cavila sobre la contradicción inherente a la grabación de los actos judiciales en vídeo, ya que son actos públicos, si bien se ha de contar con el beneplácito de todos los actores implicados para poder grabar (2013: 323-324). Aunque en la actualidad en España sea obligatoria la grabación de las vistas penales, laborales y administrativas, estos profesionales se muestran reticentes a facilitar tales grabaciones -incluso- a investigadores (Cervera y Torres 2015: 10). Desde Costa Rica, Quesada Pacheco (1991: 87-88) puntualiza que las grabaciones en audio de juicios penales presentan el problema de que en ocasiones el volumen utilizado por los participantes es tan bajo que no se puede entender el discurso emitido; aparte de señalar los obstáculos derivados de la comunicación no verbal, puesto que a veces los interrogados responden afirmativa o negativamente de manera gestual mediante un movimiento de cabeza. Por tales motivos, este autor delibera que -en última instancia- la transcripción de un juicio no es fiel al evento comunicativo. Además, para que este tipo de investigaciones resulten útiles, como ya advertían estudios pioneros sobre interrogatorios judiciales desde el enfoque psicológico, "[...] there be an ongoing collaboration between lawyers and social scientists to carry out the empirical investigation necessary to test such legal assumptions and practices" (Marshall, Marquis y Oskamp 1971: 1639-1640).

El presente artículo se plantea como objetivo indagar en los tipos de preguntas utilizadas en interrogatorios judiciales españoles desde un perfil cuantitativo; más en concreto, se han analizado dos corpus grabados en Almería y Barcelona respectivamente. Se toma como base la publicación de Ridao (2009) -que a su vez emana, con modificaciones parciales, 
del método investigador utilizado por Danet, Hoffman et alii (1980) y Harris (1984) principalmente-, la cual aporta datos cuantitativos de los tipos de preguntas utilizadas en juicios celebrados en el Juzgado de lo Penal de Almería capital. Esta información estadística se contrasta con los datos obtenidos tras el análisis del Corpus de Procesos Penales grabado en Barcelona (Taranilla 2012). Las cuestiones de partida de esta pesquisa son: ¿para formular una pregunta siempre debe emplearse un patrón entonativo propiamente interrogativo?, ¿se pueden establecer paralelismos entre los patrones de entonación y el rol de los actores?, ¿existe relación entre patrones entonativos empleados por el interrogador y la procedencia geográfica de los corpus? Ante tan vasto campo de estudio, en este artículo se dará respuesta a los dos primeros interrogantes, mientras que de la última se proporcionan claves para establecer futuras líneas de investigación.

\section{Estado de la cuestión}

Detallar - e incluso tan solo citar- de manera exhaustiva los trabajos que se han llevado a cabo sobre esta temática extralimita la extensión de un artículo de investigación, tanto si se analiza el ámbito anglosajón como solo el hispánico, aunque este último posea una producción bibliográfica menor. Por ende, en este modesto estudio se ha optado por hablar de tendencias generales confrontando la bibliografía en lengua española con la de lengua inglesa. Tan solo se profundizará en las publicaciones cuya temática es más afín a la del presente artículo: el análisis de las preguntas en interrogatorios judiciales.

Los estudios interdisciplinares sobre lingüística y derecho conforman un terreno investigador muy consolidado; de forma simultánea, en esta materia también han mostrado interés la psicología o la traducción, por citar tan solo algunas disciplinas que han trabajado con corpus legales. Retomando en enfoque lingüístico, se ha de señalar que, ciertamente, en el mundo del derecho el dominio de la palabra -tanto en la dimensión oral como en la escritaconstituye una clave fundamental para ejercer con éxito tal profesión. En consecuencia, son bastantes los manuales en español sobre oratoria y elocuencia forense que se han elaborado desde el siglo XIX hasta la actualidad, entre los que destacan los de Sainz de Andino (1828), Pérez de Anaya (1848-1849), Miguel y Romero (1901) o Elguero (1917), por resultar precursores en aplicar los principios de la oratoria al ámbito legal; estas publicaciones afrontan tanto el estudio del discurso escrito como el discurso oral.

Al confrontar el panorama investigador hispánico y anglosajón, se observa que la situación de este segundo difiere de manera notoria, sobre todo en términos cuantitativos. $\mathrm{Si}$, por un lado, existe una arraigada y fecunda tendencia a indagar en el lenguaje jurídico, por el otro, se ha de tener en cuenta que la mayoría de tales pesquisas aborda el análisis de corpus escritos y buena parte se centra en documentos fechados antes del siglo XIX; como es el caso en el ámbito hispánico de Perona Sánchez (1984), Porcar Miralles (1991), Díez de Revenga e Igualada Belchí (1997), Puche Lorenzo (2002) o Abad Merino (2011), por citar solo algunos ejemplos de una interminable nómina. Del mismo modo, también existe una marcada tendencia por indagar en estudios descriptivos sobre el lenguaje jurídico o administrativo; algunos trabajos de esta índole son Rodríguez Aguilera (1969), Pardo (1992), Martínez Marín (1997) o Alcaraz Varó, Hughes y Gómez (2014).

Continuando con la bibliografía en español, se aprecia que en las últimas décadas se ha mostrado un acusado interés por examinar corpus orales desde diferentes enfoques, como son: 
análisis lingüístico de pruebas testimoniales en juicios penales (Quesada 1991), la función dialógica del juez en vistas orales (Hernández Terrés 1997), los tipos de preguntas utilizadas en interrogatorios judiciales (Ridao 2009), el corpus judicial (Ridao 2010), las estrategias de (des) cortesía en juicios (Bernal 2010; Ridao 2016), la caracterización lingüístico-comunicativa del subgénero discursivo juicio oral (Briz 2011), la impersonalidad como mecanismo atenuador en los juicios orales (Villalba 2012), el discurso como narración (Taranilla 2012), el método para la confección de un corpus jurídico (Taranilla 2013), o las formas de tratamiento nominales y las fórmulas apelativas convencionalizadas (Villalba, en prensa), entre otros. Como se puede deducir, en español -según los datos manejados- no existen estudios con la naturaleza de la investigación propuesta en este artículo, con la excepción de Ridao (2009).

La producción bibliográfica en el contexto anglosajón relacionada con la lingüística forense desde sus inicios ha puesto de manifiesto el papel determinante que asumen las interrogaciones cifradas en los contextos judiciales. Desde el prisma de la psicología, el temprano trabajo de Muscio indaga en cómo la forma en que se cifra la pregunta condiciona la respuesta que emite el interrogado; exactamente afirma que "[...] the answer to a question is under certain conditions partly determined by the question's form" (1916: 351). Por su parte, la investigación de Danet, Hoffman et alii (1980) desarrolla un método empírico para realizar una descripción sistemática de la manera de interrogar en los contextos judiciales; con esta finalidad, ofrece una clasificación de las formas lingüísticas empleadas por los interrogadores judiciales para obtener la información de sus interrogados: intervenciones declarativas, preguntas cerradas, preguntas abiertas, ruegos y formas imperativas. La metodología de discriminar entre formas interrogativas y declarativas también es utilizada en Harris (1984). A esta también se une Philips (1984), con la diferencia de que este trabajo aborda detalladamente la forma en que la pregunta influye en la emisión de la respuesta; en un estudio posterior (Philips 1987) se centra en el uso de las preguntas abiertas en los juicios. En esta línea de análisis, Luchjenbroers (1997) llega a la conclusión de que los testigos suelen narrar poco sobre los hechos ocurridos en el crimen cuando esta información se le transmite al jurado, al tiempo que corrobora que las estrategias utilizadas por el abogado están destinadas a demostrar la afinidad existente entre este actor y su cliente.

Desde otro enfoque, Woodbury (1984) reflexiona sobre cómo el interrogador controla las pruebas que recibe el jurado para, a continuación, indagar en cómo adecua la información nueva y la ya proporcionada con miras a que sea emitida en el momento que considera más proclive. Así, con respecto al control de la información como estrategia en los interrogatorios judiciales, establece la siguiente clasificación con un clímax de orden ascendente del tipo de intervenciones con fines interrogativos: broad wh-questions, narrow wh-questions, alternative questions, grammatical yes/no questions, negative gramatical yes/no questions, prosodic questions y tag questions. De Drew (1992) se ha de resaltar que indague -ejemplificando con transcripciones- la hostilidad en el interrogatorio del sistema judicial angloamericano. En esta misma línea, pero en el ámbito hispánico basándose en bibliografía propia de estrategias de (des)cortesía, y también aportando transcripciones, se sustentan los estudios de Bernal (2010) y Ridao (2016). Junto a ello, en Storey-Whyte (1997), se trata el problema de la dificultad de la comprensión del lenguaje jurídico por participantes ajenos al ámbito legal; igualmente, en consonancia con otros estudios precedentes, ratifica que la utilización de preguntas cerradas tiene la finalidad de monopolizar el discurso, y por extensión, controlarlo. Desde el ángulo de la interpretación, Rigney (1999) pone de manifiesto 
la manipulación lingüística imperante en las interrogaciones como una clave estratégica de dominación del contexto legal, atendiendo a la secuencia pregunta/respuesta. En el caso de interrogatorios donde se necesita un intérprete, el control de los interrogadores sobre las declaraciones de los interrogados se ve aminorado en tanto que existe un cambio de lengua y, habitualmente, para los intérpretes la pragmática de las interrogaciones como vía de manipulación pasa desapercibida.

Si bien Heffer (2010) centra su atención en examinar cómo se construyen las historias del crimen en el juicio -tema del que también se ocupa Baffy y Marsters (2015)-, los interrogatorios son objeto de su análisis. En particular, indica que un abogado maneja dos estrategias para controlar la narración del crimen en un juicio: la primera, seleccionando a los testigos, y, la segunda, guiando a tales testigos a través del interrogatorio (2010: 205). En un estudio ulterior, Henderson, Heffer y Kebbell señalan que el principal problema que presenta un interrogatorio es el empleo de preguntas que sugieren la respuesta, lo que conlleva coacción; por ende, "Cross-examination almost invariably involves a high proportion of suggestive questions" (2016: 184) y en unas páginas posteriores afirman: "In addition to criticisms of its potential for contaminating and confusing evidence, research also suggests that cross-examination is ineffective in revealing unreliable witnesses" (2016: 187).

\section{Corpus}

En tanto que el lenguaje legal se considera un discurso de especialidad, resulta necesaria la confección de corpus que permitan indagar en estos contextos. Efectivamente, "[...] a corpus can be used in different ways in order to validate, exemplify or build up a language theory" (Tognini-Bonelli 2001: 65). En esta ocasión, se investiga en el perfil oral, pues se someten a estudio vistas orales en materia penal celebradas en España. Debido al carácter comparativo / contrastivo con el que ha sido concebido este estudio, se han utilizado dos corpus de juicios orales. El orden cronológico de publicación de estos corpus se ha respetado a la hora de asignar la denominación de corpus I y II.

El primero de ellos ha sido recopilado en el Juzgado de lo Penal de Almería capital entre los años 2000 y 2002. El intervalo de tiempo existente entre la fecha de la recogida del corpus y la actualidad no es casual, sino que se justifica por la preservación de la intimidad de los participantes. El cómputo total es de quince juicios cuya temática se centra en robo, robo con intimidación, delito de lesiones y amenazas, denuncia falsa, delito contra el deber de cumplir la prestación social sustitutoria, agresión, apropiación indebida y malos tratos. En cuanto a la duración, todos los juicios analizados suman tres horas, diecisiete minutos y nueve segundos. Los datos sobre el tipo de preguntas existentes en tales juicios han sido analizados en Ridao (2009); la mayor parte de los materiales sobre los que se han efectuado tal estudio ha sido publicada en Ridao (2010).

El segundo corpus se ha grabado en los Juzgados de lo Penal de la ciudad de Barcelona entre febrero de 2009 y julio de 2010. Se trata de diez juicios celebrados con motivo de robo con violencia más lesiones, quebrantamiento de condena, lesiones, simulación de delito, robo con fuerza, amenazas e injurias, delito contra la seguridad vial, tenencia ilícita de armas, delito contra la propiedad intelectual y robo con violencia. La duración de todos estos juicios es exactamente cinco horas, cincuenta y nueve minutos y diecinueve segundos. La transcripción de dicho corpus está disponible en Taranilla (2012). 
En ambos corpus, se han examinado únicamente las intervenciones destinadas a interrogar a los testigos; es decir, los turnos de habla asumidos por el juez, el fiscal o el letrado con el fin de extraer información de los interrogados. En total se han analizado 2197 intervenciones, de las cuales 995 pertenecen al corpus I y 1202 al corpus II. Por tanto, aunque la duración total de los corpus difiere, ambos presentan el denominador común de estar compuestos por un número similar de intervenciones.

\section{Método}

El análisis aplicado en este artículo emplea como base la metodología de investigación ya utilizada en Danet, Hoffman et alii (1980) y Harris (1984), si bien se ha adaptado a las características propias de los corpus españoles; otros estudios que usan modelos ligeramente diferentes son Philips (1984, 1987), Woodbury (1984) o Luchjenbroers (1997). El hecho de que en español no exista -según los datos manejados- estudios desde este enfoque, excepto Ridao (2009), justifica la utilización de una forma de análisis con tan dilatada trayectoria en el ámbito anglosajón. En particular, el método seguido ha consistido en clasificar las intervenciones con fines interrogadores en tres categorías en función de su patrón entonativo ${ }^{1}$ e intención pragmática:

- Las interrogaciones tradicionales son aquellas que la gramática tradicional caracteriza con una entonación propiamente interrogativa; esto es, el fundamental correspondiente con su entonación se ajusta -en mayor o menor medida- al prototipo de entonación interrogativa; en consecuencia, poseen una entonación ascendente al comienzo y al final del fundamental. En el plano escrito estos enunciados están encabezados por los signos de interrogación de apertura y finalizados por los símbolos de interrogación de cierre. He aquí dos ejemplos extraídos respectivamente de los corpus I y II.

(1) 31- Letrada: ¿había usted ingerido grandes cantidades deee pastillas como Tranxilium y bebidas alcohólicas? § [Corpus I, juicio 2]

(2) AD5: ¿entonces / qué participación tuvo este señor en- / en los hechos? / ¿qué fue lo que ocurrió? / [Corpus II, juicio 3]

- Las interrogaciones pragmáticas son aquellas que presentan una entonación prototípicamente enunciativa; sin embargo, la intención ilocutiva ${ }^{2}$ desde la perspectiva pragmática se centra en que el interrogado responda al tema que el interrogador está tratando. En realidad, en la mayor parte de estas intervenciones se narran hechos, de tal manera que es el interrogador quien suele monopolizar el discurso y con asiduidad

1 A colación de esta cuestión, resulta necesario tener en cuenta que: "El suprasegmento entonativo es uno de los componentes más complejos de una lengua: sus variadas formas, sus diversas funciones, su incidencia más o menos directa en la significación y en la caracterización del hablante lo convierten en un campo de estudio en el que no faltan las dificultades" (Quilis, 1993: 409).

2 Aunque en la actualidad este concepto pragmático esté muy extendido, se quiere aclarar que ha sido tomado de Austin (1962). 
se pueden considerar actos de habla perlocutivos ${ }^{3}$, dado que el interrogador influye notoriamente en el discurso emitido por el interrogado. Estos enunciados carecen de signos de interrogación en el plano escrito, como consecuencia de que en la dimensión oral el fundamental responde a un prototipo entonativo enunciativo. Seguidamente, se muestran fragmentos de las transcripciones que corroboran este razonamiento:

\section{(3) 471- Letrado: (( ))] COMO ESTABA USTED AHÍ A LO MEJOR ESCUCHABA LAS} CONVERSACIONES $\S$

472- Testigo 3: el tiempo que yo estuve allí ya no se daban clases / allí ya se estaba recogiendo eeel / el material §

473- Letrado: ya [pero

474- Testigo 3: allí] no llegaba nadie a que le dieran clase $\S$

475- Letrado: pero nadie iba a quejarse /

476- Testigo 3: yo no oí a nadie ir a quejarse $\S$

477- Letrado: solo a doña \{nombre de la acusadora\} § [Corpus I, juicio 10]

(4) J25: ah / le cogió / porque acababa de pasar eso / y pensó que usted iba con [la chica / muy bien] /

ACU227: [(xxx) ¿vale?]

J26: le dijo que le había robado / entonces /

ACU28: sí / entonces la señora Rita/ Gómez / me- / le dijo / "suéltalo" / me soltó / vinieron los mossos d'esquadra / e:: / me pidieron el dni / todo / y bueno / (xxx) / vinieron todoos / J27: ya / [Corpus II, juicio 10]

- Las interrogaciones hibridas son aquellas que combinan las interrogaciones tradicionales con las interrogaciones pragmáticas. A diferencia de las interrogaciones pragmáticas y en consonancia con las tradicionales, en las híbridas sí hay patrones interrogativos, pero solo en parte del enunciado, que normalmente es al final; o sea, que el fundamental al principio se suele caracterizar por responder al prototipo enunciativo y después se identifica con el interrogativo, y dicha situación en el plano escrito se corresponde con la colocación del signo de apertura de interrogación ya avanzado el enunciado y el signo de cierre de la interrogación al final del enunciado. En estas intervenciones se presentan ejemplos de interrogaciones híbridas:

(5) 10- Fiscal: y tenía que cumplir la prestación social sustitutoria en la asociación \{nombre de la asociación $\}^{\circ}\left(\right.$ en $\{\text { nombre de un pueblo\} })^{\circ} /$ ¿es cierto que se incorporó usted y a los quince días abandonó? §

11- Acusado: a los quince días no / al mes $\S$

12- Fiscal: nooo ((casi al mes)) / ¿se incorporó usted concretamente el día dieciséis del seis del noventa y nueve y el seis del siete del noventa y nueve lo dejó? ¿es cierto eso? $\S[$ Corpus I, juicio 6]

(6) MF21: entonces dice usted / que cogió la pistola / para quitarse de en medio / e:: / es decir / que la persona que le ofreció la pistola / ¿usted estuvo de acuerdo? / ¿usted le [pidió la pistola? /] [Corpus II, juicio 8] 


\section{Análisis y resultados}

Haciendo estadísticas generales - esto es, sin distinguir en función de actores ni de corpus-, se observa el predominio de la interrogación tradicional $(44,3 \%)$, seguida de la interrogación pragmática $(32,8 \%)$ y en último lugar se halla la interrogación híbrida $(22,8$ $\%$ ). Hay diferencias muy significativas en los datos concretos de cada corpus, cuestión que se tratará detalladamente más adelante.

\begin{tabular}{|c|c|c|c|c|c|c|}
\hline \multirow{2}{*}{$\begin{array}{c}\text { Tipos de } \\
\text { entonación }\end{array}$} & $\begin{array}{c}\text { Frecuencia } \\
\text { absoluta }\end{array}$ & $\begin{array}{c}\text { Frecuencia } \\
\%\end{array}$ & $\begin{array}{c}\text { Frecuencia } \\
\text { absoluta }\end{array}$ & $\begin{array}{c}\text { Frecuencia } \\
\%\end{array}$ & $\begin{array}{c}\text { Frecuencia } \\
\text { absoluta }\end{array}$ & $\begin{array}{c}\text { Frecuencia } \\
\%\end{array}$ \\
\hline $\begin{array}{c}\text { Interrogación } \\
\text { tradicional }\end{array}$ & 232 & $23,3 \%$ & 742 & $61,7 \%$ & 974 & $\mathbf{4 4 , 3} \%$ \\
\hline $\begin{array}{c}\text { Interrogación } \\
\text { pragmática }\end{array}$ & 437 & $43,9 \%$ & 285 & $23,7 \%$ & 722 & $\mathbf{3 2 , 8} \%$ \\
\hline $\begin{array}{c}\text { Interrogación } \\
\text { híbrida }\end{array}$ & 326 & $32,7 \%$ & 175 & $14,5 \%$ & 501 & $\mathbf{2 2 , 8} \%$ \\
\hline Total & 995 & $\mathbf{1 0 0 \%}$ & $\mathbf{1 2 0 2}$ & $\mathbf{1 0 0} \%$ & $\mathbf{2 1 9 7}$ & $\mathbf{1 0 0 \%}$ \\
\hline
\end{tabular}

Tabla 1: Frecuencia de los tipos de enunciados.

A continuación, se desglosan los datos en función de los actores que emiten las intervenciones. En primer lugar, se muestra la información relativa a la figura del juez. Las estadísticas de ambos corpus señalan que la interrogación tradicional es la más utilizada $(56,9$ \%) por este actor y tras ella se sitúan la interrogación pragmática (24\%) y la interrogación híbrida (18,9\%). Se aprecian grandes divergencias al comparar los datos de ambos corpus por separado, pues en el corpus I el porcentaje más alto lo asume la interrogación híbrida (56\%), seguida de la interrogación tradicional (31,8\%), y, por último, con una cifra bastante baja la interrogación pragmática $(12,1 \%)$; en contraposición, en el corpus II predomina la interrogación tradicional (61\%), adquiriendo menor incidencia la interrogación pragmática $(25,9 \%)$ y la interrogación híbrida $(13 \%)$.

\begin{tabular}{|c|c|c|c|c|c|c|}
\hline \multirow{2}{*}{$\begin{array}{c}\text { Tipos de } \\
\text { enunciados }\end{array}$} & $\begin{array}{c}\text { Corpus I } \\
\text { Frecuencia } \\
\text { absoluta }\end{array}$ & $\begin{array}{c}\text { Frecuencia } \\
\%\end{array}$ & $\begin{array}{c}\text { Frecuencia } \\
\text { absoluta }\end{array}$ & $\begin{array}{c}\text { Frecuencia } \\
\%\end{array}$ & $\begin{array}{c}\text { Frecuencia } \\
\text { absoluta }\end{array}$ & $\begin{array}{c}\text { Frecuencia } \\
\%\end{array}$ \\
\hline $\begin{array}{c}\text { Interrogación } \\
\text { tradicional }\end{array}$ & 21 & $31,8 \%$ & 252 & $61 \%$ & 273 & $\mathbf{5 6 , 9 \%}$ \\
\hline $\begin{array}{c}\text { Interrogación } \\
\text { pragmática }\end{array}$ & 8 & $12,1 \%$ & 107 & $25,9 \%$ & 115 & $\mathbf{2 4 \%}$ \\
\hline $\begin{array}{c}\text { Interrogación } \\
\text { híbrida }\end{array}$ & 37 & $56 \%$ & 54 & $13 \%$ & 91 & $\mathbf{1 8 9} \%$ \\
\hline Total & $\mathbf{6 6}$ & $\mathbf{1 0 0 \%}$ & 413 & $\mathbf{1 0 0 \%}$ & 479 & $\mathbf{1 0 0 \%}$ \\
\hline
\end{tabular}

Tabla 2: Frecuencia de los tipos de enunciados en la figura del juez. 
En el caso de la figura del fiscal, los números recogidos en los dos corpus señalan que se decanta en mayor medida por el uso de la interrogación tradicional $(41,5 \%)$, seguida de la interrogación pragmática (33,8 \%) y la interrogación híbrida (24,5 \%). Una vez más, se hallan diferencias muy significativas al contrastar los dos corpus. La interrogación pragmática obtiene la cifra más alta $(42,6 \%)$, la interrogación híbrida se ubica en una segunda posición $(35,9 \%)$ y la interrogación tradicional es la menos utilizada $(21,4 \%)$ en el corpus I; por orden descendente en el corpus II se aprecia un marcado predominio de la interrogación tradicional (61,1\%), frente a un bajo empleo de la interrogación pragmática $(25,3 \%)$ y de la interrogación híbrida $(13,5 \%)$.

\begin{tabular}{|c|c|c|c|c|c|c|}
\hline \multirow{2}{*}{$\begin{array}{c}\text { Tipos de } \\
\text { enunciados }\end{array}$} & \multicolumn{2}{|c|}{ Corpus I } & \multicolumn{2}{c|}{ Corpus II } & \multicolumn{2}{c|}{ Ambos corpus } \\
\cline { 2 - 7 } & $\begin{array}{c}\text { Frecuencia } \\
\text { absoluta }\end{array}$ & $\begin{array}{c}\text { Frecuencia } \\
\%\end{array}$ & $\begin{array}{c}\text { Frecuencia } \\
\text { absoluta }\end{array}$ & $\begin{array}{c}\text { Frecuencia } \\
\%\end{array}$ & $\begin{array}{c}\text { Frecuencia } \\
\text { absoluta }\end{array}$ & $\begin{array}{c}\text { Frecuencia } \\
\%\end{array}$ \\
\hline $\begin{array}{c}\text { Interrogación } \\
\text { tradicional }\end{array}$ & 86 & $21,4 \%$ & 253 & $61,1 \%$ & 339 & $\mathbf{4 1 , 5 \%}$ \\
\hline $\begin{array}{c}\text { Interrogación } \\
\text { pragmática }\end{array}$ & 171 & $42,6 \%$ & 105 & $25,3 \%$ & 276 & $\mathbf{3 3 , 8} \%$ \\
\hline $\begin{array}{c}\text { Interrogación } \\
\text { híbrida }\end{array}$ & 144 & $35,9 \%$ & 56 & $13,5 \%$ & 200 & $\mathbf{2 4 , 5} \%$ \\
\hline Total & 401 & $\mathbf{1 0 0} \%$ & 414 & $\mathbf{1 0 0} \%$ & $\mathbf{8 1 5}$ & $\mathbf{1 0 0 \%}$ \\
\hline
\end{tabular}

Tabla 3: Frecuencia de los tipos de enunciados en la figura del fiscal.

Las cifras totales de los dos corpus demuestran que el letrado opta en mayor número de ocasiones por el empleo de la interrogación tradicional (40\%), seguida de la interrogación pragmática $(36,6 \%)$ y la interrogación híbrida $(23,2 \%)$. Como en los casos anteriores, los resultados obtenidos en los dos corpus muestran diferentes tendencias: en el corpus I destaca la interrogación pragmática $(48,8 \%)$, con una prevalencia inferior aparece la interrogación híbrida $(27,4 \%)$ y la interrogación tradicional se ubica en última posición $(23,6 \%)$; en el corpus II es la interrogación tradicional la más recurrida (63,2 \%), presentando las otras dos tipologías unos índices de frecuencia notoriamente más bajos -interrogación pragmática $(19,4 \%)$ e interrogación híbrida $(17,3 \%)-$.

\begin{tabular}{|c|c|c|c|c|c|c|}
\hline \multirow{2}{*}{$\begin{array}{c}\text { Tipos de } \\
\text { enunciados }\end{array}$} & \multicolumn{2}{|c|}{ Corpus I } & \multicolumn{2}{c|}{ Corpus II } & \multicolumn{2}{c|}{ Ambos corpus } \\
\cline { 2 - 7 } & $\begin{array}{c}\text { Frecuencia } \\
\text { absoluta }\end{array}$ & $\begin{array}{c}\text { Frecuencia } \\
\%\end{array}$ & $\begin{array}{c}\text { Frecuencia } \\
\text { absoluta }\end{array}$ & $\begin{array}{c}\text { Frecuencia } \\
\%\end{array}$ & $\begin{array}{c}\text { Frecuencia } \\
\text { absoluta }\end{array}$ & $\begin{array}{c}\text { Frecuencia } \\
\%\end{array}$ \\
\hline $\begin{array}{c}\text { Interrogación } \\
\text { tradicional }\end{array}$ & 125 & $23,6 \%$ & 237 & $63,2 \%$ & 362 & $\mathbf{4 0 \%}$ \\
\hline $\begin{array}{c}\text { Interrogación } \\
\text { pragmática }\end{array}$ & 258 & $48,8 \%$ & 73 & $19,4 \%$ & 331 & $\mathbf{3 6 , 6} \%$ \\
\hline $\begin{array}{c}\text { Interrogación } \\
\text { híbrida }\end{array}$ & 145 & $27,4 \%$ & 65 & $17,3 \%$ & 210 & $\mathbf{2 3 , 2} \%$ \\
\hline Total & $\mathbf{5 2 8}$ & $\mathbf{1 0 0} \%$ & $\mathbf{3 7 5}$ & $\mathbf{1 0 0} \%$ & $\mathbf{9 0 3}$ & $\mathbf{1 0 0 \%}$ \\
\hline
\end{tabular}

Tabla 4: Frecuencia de los tipos de enunciados en la figura del letrado. 


\section{Discusión}

Para valorar correctamente los resultados obtenidos en esta investigación, se debe indicar el número de intervenciones que ha asumido cada actor. En esta cuestión, hay que señalar que en el corpus II la figura del juez adquiere un rol bastante más activo que en el corpus I, pues en el primer caso tan solo emite 21 intervenciones con fines interrogatorios, mientras que en el segundo la cifra suma las 252; el resto de intervenciones las dedica a presentar el juicio y establecer los turnos de habla, por lo que no tiene cabida su estudio en este análisis. A propósito de esta cuestión, conviene recordar que, tal como señala Hernández Terrés (1997: 43-44), las competencias del juez se ajustan a: (1) iniciar el acto; (2) dar la palabra a cada uno de los intervinientes; (3) corregir el modo y la calidad de las intervenciones; (4) exhortar a decir verdad al acusado; (5) tomar juramento o promesa de veracidad a los testigos y peritos; (6) valorar la pertinencia o no de las intervenciones, y en este sentido permitirlas o no; y (7) exigir que las intervenciones sean lo suficientemente informativas para que pueda darse el esclarecimiento de los hechos (o sea, vela por el cumplimiento de las máximas de cantidad, cualidad, relación y manera ${ }^{4}$ ). Si bien el desglose pormenorizado en función de los corpus puede consultarse en las tablas 2, 3 y 4, he aquí un gráfico con las cifras globales:

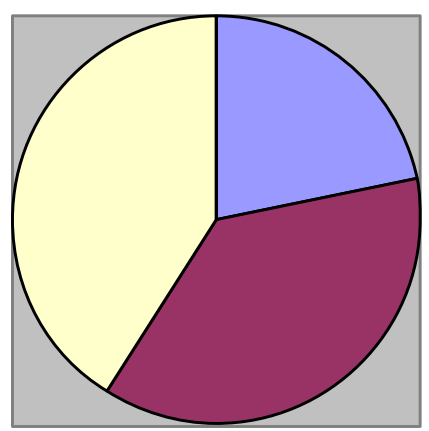

\section{$\square$ Juez: 479 \\ $\square$ Fis cal: 815 \\ $\square$ Letrado: 903 \\ Total: 2197}

Gráfico 1: Frecuencia absoluta de intervenciones en función de actores.

Comenzando con los datos globales sin distinguir actores, cabe destacar la marcada diferencia existente en la frecuencia de uso de los tipos de entonación. De esta manera, en el corpus I, el porcentaje más alto se sitúa en la interrogación pragmática $(43,9 \%)$, a continuación está la interrogación híbrida $(32,7 \%)$ y en último lugar se localiza la interrogación tradicional (23, $3 \%$ ); en contraposición, el corpus II se caracteriza por un elevado porcentaje en el empleo de la interrogación tradicional $(61,7 \%)$, presentando números muy inferiores en la interrogación pragmática $(23,7 \%)$ y en la interrogación híbrida (14,5\%). El siguiente gráfico ilustra de manera visual la información aportada en el presente estudio:

4 Máximas acuñadas por Grice (1991[1975]). 

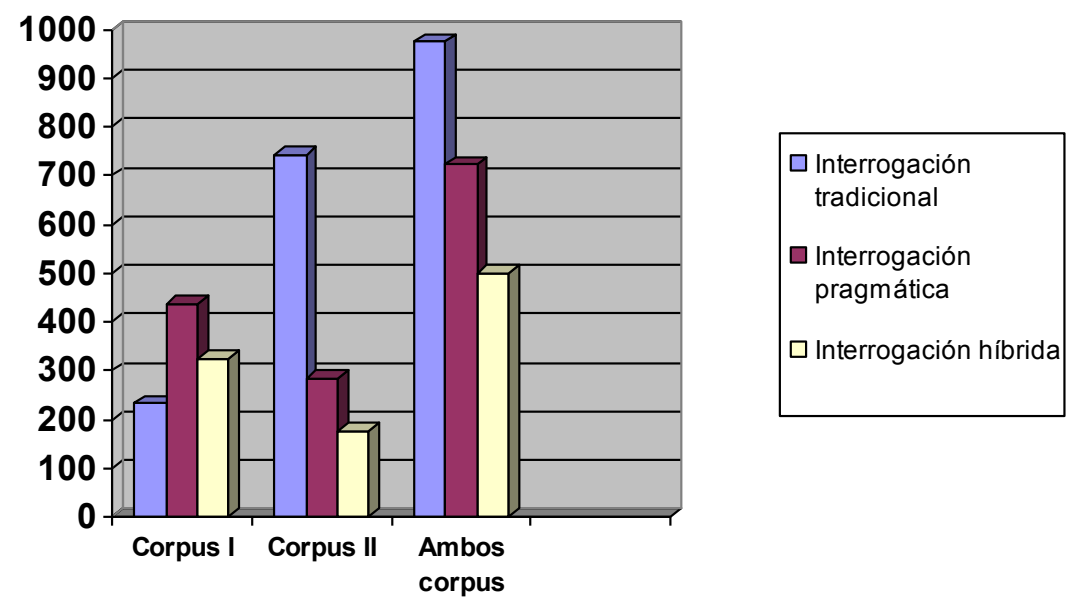

Gráfico 2: Frecuencia absoluta en función de los tipos de entonación.

Si se toman en consideración los datos obtenidos en la suma de ambos corpus, los tres actores analizados -juez, fiscal y letrado- muestran una utilización más elevada de interrogaciones tradicionales $(56,9 \%, 41,5 \%$ y $40 \%$, respectivamente), en segundo lugar se encuentra la interrogación tradicional $(24 \%, 33,8 \%$ y 36,6 \%) y, por último, la interrogación híbrida (18,9\%. 24,5\% y 23,2\%). Sin embargo, las grandes discrepancias se ponen de manifiesto al analizar los datos particulares de cada corpus.

Los resultados publicados en Danet, Hoffman et alii (1980) presentan semejanzas con los obtenidos en el corpus II; si bien estos autores no aportan datos estadísticos globales, se observan porcentajes más altos en los distintos actores en enunciados interrogativos que en declarativos. Además, en la citada investigación se distingue entre direct, cross y combined examination ${ }^{5}$, parámetros que no se han tomado en consideración en el presente artículo porque se ha considerado más relevante analizar únicamente el rol del participante. En sintonía con tales datos se encuentran los resultados hallados en Harris (1984), pesquisa que sí aporta información estadística global de un corpus bastante limitado pues en total se han analizado 188 intervenciones: las intervenciones interrogativas ascienden al $69.1 \%$, las declarativas al $23.9 \%$ y moodless $^{6}$ al $6.9 \%$.

Los gráficos que se exponen a continuación señalan las divergencias existentes en el empleo de las distintas tipologías de entonación utilizadas en los interrogatorios sometidos a examen. A continuación, se especifican las diferencias entre los dos corpus:

En el corpus I las elecciones lingüísticas efectuadas por el juez difieren de los otros actores que también asumen el rol de interrogadores; en particular, el juez se decanta -con orden de preferencia descendente- por la interrogación híbrida (37), la interrogación tradicional (21) y la interrogación pragmática (8). Por otro lado, tanto el fiscal como el letrado utilizan

5 Clasificación cuya base se sustenta en función de si interrogador e interrogado pertenecen o no al mismo bloque (defensor o acusador).

6 Terminología utilizada por Harris (1984) para aludir a los enunciados caracterizados por un nivel o tono ascendente. 


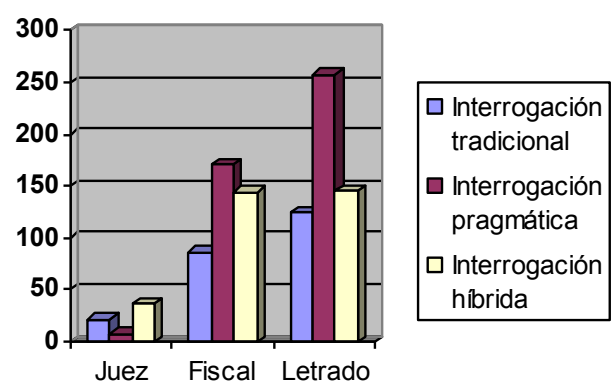

Gráfico 3:

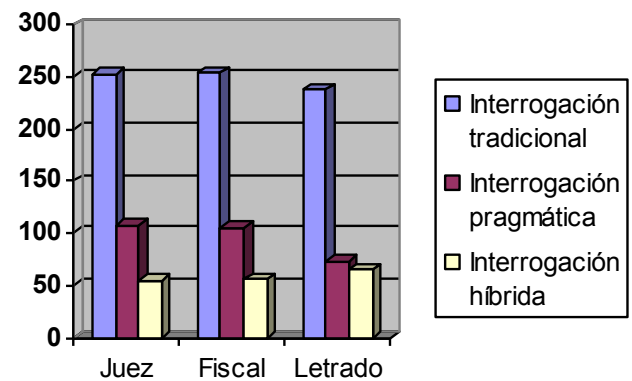

Gráfico 4:

Frecuencia absoluta del corpus II.

la interrogación pragmática con mayor asiduidad (171 y 258 respectivamente), la interrogación híbrida se coloca en una segunda posición (144 y 145), de manera que la menos usada es la interrogación tradicional ( 86 y 125).

En contraposición, los datos que se desprenden del corpus II demuestran unanimidad en cuanto al empleo de las distintas tipologías de entonación, puesto que tales preferencias son comunes a los tres actores estudiados. De esta manera, las interrogaciones tradicionales presentan cifras más altas en juez, fiscal y letrado (252, 253 y 237 respectivamente), seguida de la interrogación tradicional $(107,105$ y 73), y, en último lugar, se posiciona la interrogación híbrida (54, 56 y 65).

Tal vez las discrepancias encontradas en ambos corpus se justifiquen por el hecho de que en el ámbito del derecho se ponen de manifiesto considerablemente las divergencias existentes entre territorios y culturas, lo que implica que sea necesaria una cuidadosa reflexión sobre cómo han de confeccionarse los corpus de esta naturaleza, así como “[...] su empleo como fundamento empírico de los estudios sobre español jurídico [que] mantenga ciertas prevenciones acerca de lo que se viene practicando en otras tradiciones lingüísticas" (Taranilla 2013: 313). En realidad, las transcripciones aquí analizadas se enmarcan en el sistema judicial español, si bien en las investigaciones sobre los modelos entonativos con que se cifran los interrogatorios desde la perspectiva lingüística se han puesto de manifiesto bastantes divergencias que pueden tener una explicación en las variaciones diatópicas.

\section{Conclusiones y futuras líneas de investigación}

El estado de la cuestión sobre el lenguaje legal, en general, tanto en el panorama hispano como en el anglosajón se ha caracterizado por un predominio de estudios sustentados sobre corpus escritos, si bien en los últimos años están apareciendo trabajos cuyos corpus poseen naturaleza oral. La bibliografía inglesa no solo asume el nacimiento de las pioneras pesquisas de esta índole, sino que también presenta cuantitativamente cifras más elevadas de publicaciones. Al tratarse de un campo con carácter interdisciplinar, en el seno de la lingüística forense también se adscriben trabajos propios de la psicología o la traducción. Se puede considerar que el punto del que parten las investigaciones -independientemente del enfoque que asuman- circunscritas a los interrogatorios judiciales se 
guían por la máxima de que la manera de formular la pregunta influye sustancialmente en la respuesta recibida, como consecuencia del interés del interrogador por controlar la narración de los hechos.

Del análisis de los corpus examinados en el presente artículo se deduce que en los interrogatorios judiciales la pragmática juega un papel determinante, en tanto que los enunciados con fines interrogativos no siempre se caracterizan -en términos fonéticospor asumir unos patrones entonativos propios de las interrogaciones (interrogaciones tradicionales), sino que se dan casos en los que el enunciado con fin interrogador muestra un prototipo entonativo aseverativo (interrogaciones pragmáticas) o una mezcla de ambos (interrogaciones híbridas). Esta clasificación se ha utilizado como parámetro para efectuar el análisis, así como también se ha tenido en cuenta el rol asumido por cada uno de los participantes.

Los resultados globales obtenidos en esta investigación demuestran la existencia de un mayor uso de la interrogación tradicional, en segundo lugar se situaría el empleo de la interrogación pragmática, y, por último, el de la interrogación híbrida. Sin embargo, al analizar los corpus por separado se aprecian diferencias muy significativas: en el primer corpus, predomina la interrogación pragmática, seguida de la interrogación híbrida y la interrogación tradicional; en contraposición, en el segundo corpus la interrogación tradicional es la que prevalece, a continuación le sucede por orden de frecuencia la interrogación pragmática y la interrogación híbrida. Los datos extraídos del corpus II presentan mayor afinidad con los resultados de Danet, Hoffman et alii (1980) y Harris (1984). Por la limitada extensión de los corpus analizados, tanto en español como en inglés, serían convenientes análisis de esta naturaleza con cambios en las variables diatópicas e incluso temáticas (habida cuenta de que en el presente artículo se han abordado solo casos penales).

Siguiendo con más futuras líneas de investigación, resultan necesarios estudios de esta índole enfocados desde el perfil cualitativo; es decir, aportando fragmentos de transcripción, en sintonía con los ya presentados en el apartado de Método, tal como están trabajando Cervera y Torres desde la Universidad Complutense de Madrid. Del mismo modo, publicaciones posteriores deberían indagar en el plano cuantitativo en la frecuencia de uso del tipo de preguntas abiertas formuladas en los juicios; si bien es cierto que Ridao (2009) efectúa un análisis de esas características, se trata de un trabajo muy limitado que debería completarse con otros corpus. Desde un enfoque parcialmente diferente, se precisan investigaciones en español que aborden el estudio de las respuestas, estela iniciada en el mundo anglosajón en el pasado siglo (Philips 1984; Luchjenbroers 1997).

Además, este tipo de indagaciones puede reforzarse con análisis pormenorizados de la entonación empleando para ello programas digitales de voz, tal como se viene utilizando en las publicaciones sobre pragmática sociocultural relacionadas con los estudios sobre (des) cortesía y que, indudablemente, arrojan luz sobre la problemática de la comunicación (Hidalgo y Cabedo 2014; Devís-Herraiz 2015). Es más, hay que valorar la existencia de una relación indisociable entre los elementos fónicos y la interpretación correcta del mensaje, " [...] pues vemos que la interpretación no solo depende del contexto, sino que lo paraverbal aporta un buen número de elementos interpretativos tanto para el hablante destinatario de una situación real de habla, como para el analista dedicado al estudio de la interacción hablada" (Hernández Flores 2012: 564). 


\section{Referencias bibliográficas}

Abad Merino, M. (2011). "La traducción de cartas árabes en un pleito granadino del siglo XVI. El fenómeno del romanceado como acto judicial: Juan Rodríguez y Alonso del Castillo ante un mismo documento", Al-qantara: Revista de Estudios Árabes, 32, 2, pp. 481-518.

Alcaraz Varó, E., Hughes, B. y Gómez, A. (2014). El español jurídico. Barcelona: Ariel Derecho.

Austin, J. (1962). How to do things with words. Cambridge: Harvard University Press.

Baffy, M. y Marsters, A. (2015). "The constructed voice in courtroom cross-examination", The International Journal of Speech, Language and the Law, 22, 2, pp. 143-165.

Bernal, M. (2010). "Descortesía en el contexto judicial: el caso del 11-M". En Orletti, F. y L. Mariottini (eds.). (Des)cortesía en español. Espacios teóricos y metodológicos para su estudio. RomaEstocolmo: Università degli Studi, pp. 599-636.

Briz, A. (1998). El español coloquial en la conversación. Esbozo de pragmagramática. Barcelona: Ariel.

Briz, A. (2011). "Los juicios orales: Un subgénero de lo +formal". En Fant, F. y A. M. Harvey (eds.). El diálogo oral en el mundo hispanohablante. Madrid/Frankfurt: Vervuert, pp. 139-162.

Cervantes, M. de (1605). El Ingenioso Hidalgo don Quijote de la Mancha. Madrid: Imprenta Iuan de la Cuefta.

Cervera, Á. y Torres, J. (2015). "Análisis conversacional del Discurso Procesal Penal”, Pragmalingüistica, 23, pp. 8-21.

Danet, B., Hoffman, K. et alii (1980). “An ethnography of questioning in the courtroom”. En Shuy, R. y A. Shnukal (eds.). Language use and the uses of language. Washington: Georgetown University Press, pp. 222-234.

Devís-Herraiz, E. (2015). "Contextos para una aplicación didáctica de la entonación atenuadora en español”, RILCE, 31, 1, pp. 154-170.

Díez de Revenga, P. e Igualada Belchí, M. D. (1997). "La lengua de los testamentos (siglos XV y XVI)", Revista de Investigación Lingüistica, 1, 1, pp. 37-58.

Drew, P. (1992). "Contested evidence in courtroom cross-examination: The case of a trial for rape". En Drew, P. y J. Heritage (eds.). Talk at work: Interaction in institutional settings. Cambridge: Cambridge University Press, pp. 470-520.

Elguero, F. (1917). Lecciones de elocuencia forense. México: Manuel León Sánchez.

Grice, P. (1991[1975]). “Lógica y conversación”. En Valdés, L. (ed.). La búsqueda del significado. Murcia: Tecnos, pp. 511-530.

Harris, S. (1984). "Questions as a mode of control in magistrates' courts", International Journal of the Sociology of Language, 49, pp. 5-27.

Heffer, C. (2005). The language of jury trials: A corpus-aided analysis of legal-lay discourse. Basingstoke/Nueva York: Palgrave.

Heffer, C. (2010). "Narrative in the trial". En Coulthard, M. y A. Johnson (eds.). The Routledge Handbook of Forensic Linguistics. Nueva York: Routledge, pp. 199-217.

Henderson, E., Heffer, C. y Kebbell, M. (2016). "Courtroom questioning and discourse". En Oxburgh, G., T. Myklebust et alii (eds.). Communication in investigative and legal contexts: Integrated approaches from Forensic Pshychology, Linguistics and Law Enforcement. Oxford: WileyBlackwell, pp. 181-208.

Hernández Flores, N. (2012). "Presencia, relevancia y perspectivas del elemento fónico en estudios de (des)cortesía”. En Escamilla Morales, J. y G. Henry Vega (eds.). Miradas multidisciplinares a los fenómenos de cortesía y descortesía en el mundo hispánico. Estocolmo: Servicio de Publicaciones de la Universidad de Estocolmo, pp. 545-567.

Hernández Terrés, J. M. (1997). "Particularidades lingüísticas del acto del juicio oral: La función dialógica del juez”. En AA. VV. (eds.). Panorama de la investigación lingüística a l'Estat Espanyol: Actes del I Congrés de Lingüistica General. Valencia: Universidad de Valencia, pp. 42-48. 
Hidalgo, A. y Cabedo, A. (2014). "On the importance of the prosodic component in the expression of linguistic im/politeness", Journal of Politeness Research, 10, 1, pp. 5-27.

Luchjenbroers, J. (1997). "In your own words...: Questions and answers in a Supreme Court trial", Journal of Pragmatics, 27, pp. 477-503.

Marshall, J., Marquis, K. y Oskamp, S. (1971). "Effects of kind of question and atmosphere of interrogation on accuracy and completeness of testimony", Harvard Law Review, 84, pp. 1620-1643.

Martínez Marín, J. (1997). "El lenguaje administrativo: Descripción y norma”, Lingüística Español Actual, XIX, 2, pp. 215-228.

Miguel y Romero, M. (1901). Lecciones y modelos de práctica forense. Valladolid: Andrés Martín.

Muscio, B. (1916). "The influence of the form of the question", British Journal of Psychology, 8, pp. 351389.

Pardo, M. L. (1992). Derecho y lingüistica. Cómo se juzga con palabras. Análisis lingüístico de sentencias judiciales. Buenos Aires: América Latina Centro Editor.

Pérez de Anaya, F. (1848-1849). Lecciones y modelos de elocuencia forense. Madrid: Imprenta de Don Baltasar González.

Perona Sánchez, J. (1984). Lengua general y lengua jurídica en el Fuero de Alba de Tormes. Murcia: Universidad de Murcia.

Philips, S. (1984). "The social organization of questions and answers in courtroom discourse: A study of changes of plea in an Arizona court", Text, 4, 1-3, pp. 225-248.

Philips, S. (1987). "On the use of WH questions in American courtroom discourse: A study of the relation between language form and language function". En Kedar, L. (ed.). Power thought language. Norwood: Ablex, pp. 83-112.

Porcar Miralles, M. (1991). "Los esquemas verbales hipotéticos en textos notariales (ss. XIII-XV): Diferencias y similitudes entre navarro y aragonés", Príncipe de Vergara, LII, pp. 225-239.

Puche Lorenzo, M. Á. (2002). El español del siglo XVI en textos notariales. Murcia: Universidad de Murcia.

Quesada Pacheco, J. A. (1991). “Análisis de las pruebas testimoniales en juicios penales", Revista de Ciencias Jurídicas, 68, pp. 83-95.

Quilis, A. (1993). Tratado de fonología y fonética españolas. Madrid: Gredos.

RAE-ASALE (2014). Diccionario de la Lengua Española. Madrid: Espasa Libros.

Ridao, S. (2009). "El interrogatorio en los contextos judiciales. Análisis del tipo y la frecuencia de las preguntas utilizadas en los Juzgados de lo Penal de Almería capital”, Interlingüística, 18, pp. 940-950.

Ridao, S. (2010). El género judicial. Materiales para su estudio lingüístico. Extremadura: Junta de Extremadura.

Ridao, S. (2016). "Between truth and lies: (Im)politeness strategies in trials", Revista de Llengua $i$ Dret, 65, pp. 59-75.

Rigney, A. (1999). "Questioning in interpreted testimony", Forensic Linguistics, 6, 1, pp. 83-108.

Rodríguez Aguilera, C. (1969). El lenguaje jurídico. Barcelona: Bosch.

Sainz de Andino, P. (1828). Elementos de elocuencia forense. Madrid: Julián Viana Razola.

Storey-Whyte, K. (1997). "KISSing the jury - advantages and limitations of the 'keep it simple' principle in the presentation of expert evidence to courts and juries", Forensic Linguistics, 4, 2, pp. 280-286.

Taranilla, R. (2012). La justicia narrante: Un estudio sobre el discurso de los hechos en el proceso penal. Madrid: Aranzadi.

Taranilla, R. (2013). "Aspectos metodológicos en la confección de un corpus jurídico. Consideraciones a propósito del Corpus de Procesos Penales", Revista de Investigación Lingüística, 16, pp. 311-341.

Tognini-Bonelli, E. (2001). Corpus Linguistics at work. Ámsterdam/Filadelfia: John Benjamins.

Villalba, C. (2012). "El valor atenuante de la impersonalidad en los juicios orales", Anuario de Lingüística Hispánica, 28, pp. 117-132.

Villalba, C. (en prensa). "Las formas de tratamiento nominales y fórmulas apelativas convencionalizadas en los juicios orales: ¿Elementos ritualizados o estrategias de atenuación?”, RILI.

Woodbury, H. (1984). "The strategic use of questions in court”, Semiotica, 48, 3-4, pp. 197-228. 


\section{Anexos:}

Símbolos de transcripción del corpus I’:

Estos símbolos de transcripción han sido establecidos por el grupo Val.Es.Co (Briz, 1998). No obstante, las características propias del corpus transcrito han requerido la incorporación de algunos símbolos.

: Cambio de hablante.

? Interlocutor no reconocido.

$\S$ Sucesión inmediata, sin pausa apreciable, entre dos emisiones de distintos hablantes.

[ Lugar donde se inicia un solapamiento o superposición.

] Final del habla simultánea.

$=$ Mantenimiento del turno de un participante en un solapamiento.

/ Pausa corta, inferior al medio segundo.

// Pausa entre medio segundo y un segundo.

// Pausa de un segundo o más.

(5”) Silencio (lapso o intervalo) de cinco segundos; se indica el número de segundos en las pausas de más de un segundo.

EN COCHE Pronunciación marcada o enfática.

(( )) Fragmento indescifrable.

((siempre)) Transcripción dudosa.

((...)) Interrupciones de la grabación o de la transcripción.

(en)tonces Reconstrucción de una unidad léxica que se ha pronunciado incompleta.

${ }^{\circ}()^{\circ}$ Fragmento pronunciado en un tono de voz más bajo, próximo al susurro.

(risas) Sonidos significativos e información no verbal.

aaa Alargamientos vocálicos.

nnn Alargamientos consonánticos.

ii !? Preguntas o exclamaciones retóricas (por ejemplo, las interrogaciones exclamativas: preguntas que no preguntan).

¿? Interrogaciones. También para los apéndices del tipo «¿no?», «¿eh?», «¿sabes?».

i! Exclamaciones. Expresiones irónicas.

Letra cursiva Reproducción e imitación de emisiones. Estilo directo, característico de los denominados relatos conversacionales.

$+($ ) + Fragmentos del discurso en los que se emplea un ritmo de habla muy acelerado. -( )- Fragmentos del discurso en los que se emplea un ritmo de habla muy pausado.

\{ \} Información confidencial. Por ejemplo, nombres y apellidos, o nombres de calles, pueblos o ciudades, números identificativos como el Documento Nacional de Identidad, entre otros. 
Símbolos de transcripción del corpus $\mathrm{II}^{8}$ :

\begin{tabular}{|c|c|}
\hline \multicolumn{2}{|c|}{ Aspectos prosódicos } \\
\hline.. & Pausa (< 1 segundo) \\
\hline$\ldots$ & Pausa larga (>1 segundo) \\
\hline 1 & Fin del grupo tonal \\
\hline- & Grupo tonal truncado \\
\hline ¿? & Interrogativa convencional \\
\hline$?$ & Inicio declarativo, final interrogativo \\
\hline palabra & Pronunciación enfática \\
\hline palabra & Pronunciación débil \\
\hline aa & Alargamiento vocálico \\
\hline pp & Alargamiento consonántico \\
\hline $\begin{array}{ll}<> \\
<>\end{array}$ & Solapamiento \\
\hline ( & Interrupción \\
\hline “" & cita de palabras ajenas \\
\hline \multicolumn{2}{|c|}{ Aspectos vocales: sonidos paralingüísticos } \\
\hline mhm, ha ha & Asentimiento \\
\hline $\mathrm{m}::, \mathrm{e}::, \mathrm{a}::$ & $\begin{array}{l}\text { Vacilación antes de } \\
\text { enunciado }\end{array}$ \\
\hline uf:: & Duda \\
\hline ntx & Desacuerdo (clic alveolar) \\
\hline \multicolumn{2}{|c|}{ Aspectos gestuales } \\
\hline (afirmación) & Gesto de afirmación con la cabeza \\
\hline (negación) & Gesto de negación con la cabeza \\
\hline \multicolumn{2}{|r|}{ Otras } \\
\hline $\mathrm{xxx}$ & Fragmento ininteligible \\
\hline$[(?)$ palabra $]$ & Fragmento incierto \\
\hline palabra & Discurso en otro idioma \\
\hline$\{$ comentario $\}$ & Comentario de la transcriptora \\
\hline \multicolumn{2}{|c|}{ Elementos de la situación comunicativa } \\
\hline$(\mathrm{J})$ & \begin{tabular}{l|l|} 
& Juez \\
\end{tabular} \\
\hline (MF) & Ministerio fiscal \\
\hline (D) & Defensor \\
\hline$(\mathrm{A}),(\mathrm{O})$ & Acusado \\
\hline (M), (M1), (R) & Testigo \\
\hline
\end{tabular}


